\title{
ASTROMETRIC AND PHOTOMETRIC UTILIZATION OF DISPERSED FRINGES FROM A SPACE INTERFEROMETRY MISSION
}

\author{
R.-D. SCHOLZ ${ }^{1}$, S. HIRTE ${ }^{1}$, U. BASTIAN ${ }^{2}$, S. RÖSER ${ }^{2}$ \\ 1 Astrophysikalisches Institut, An der Sternwarte 16, 14482 Potsdam, Germany \\ ${ }^{2}$ Astronomisches Rechen-Institut, Mönchhofstraße 12-14, 69120 Heidelberg, Germany
}

Dispersed interferometer fringes as a new type of signal are currently under discussion for several space interferometry mission projects (GAIA, FAME, DIVA). The dispersed fringes are directly recorded by CCD mosaics which are operated in drift-scan mode on board a continuously rotating satellite. They allow combined astrometric (perpendicular to the fringes, i.e. in the direction of the drift scan) and spectro-photometric measurements (in the direction of the dispersion of the fringes, i.e. perpendicular to the drift scan). We have simulated polychromatic dispersed fringe patterns of stars with known spectra. The specific instrument parameters used for the simulation are those envisaged for the DIVA instrument, with realistic assumptions on various noise contributions. The results can be scaled to GAIA and FAME instrument parameters. We have investigated simple algorithms which can be implemented in an on-board data reduction and used for the real-time attitude determination with bright and single stars. On the preliminary basis of 100 simulations per star of a given magnitude and spectral type, we have obtained first estimates of the achievable accuracy of the astrometric measurements and of the location of the photometric zero point $\lambda_{0}$.

\section{THE INSTRUMENT OF THE ASTROMETRIC MISSION DIVA}

\author{
S.J. WAGNER ${ }^{1}$, W. SEIFERT ${ }^{1}$, H. MANDEL ${ }^{1}$, U. BASTIAN ${ }^{2}$, S. RÖSER ${ }^{2}$ \\ 1 Landessternwarte, Königstuhl, 69117 Heidelberg, Germany \\ ${ }^{2}$ Astronomisches Rechen-Institut, Heidelberg, Germany \\ AND \\ the DIVA team \\ see http: $/ /$ www.aip.de:8080/ ${ }^{\sim}$ dso/diva
}

As a follow-up to the most successful Hipparcos mission we have suggested a mini-satellite. The instrument DIVA is a pair of two small Fizeau interferometers with a fixed angular separation scanning the entire sky in a Hipparcos-type fashion. Despite its small apertures the very efficient optical design and the use of CCD detectors will allow a significant increase in performance compared to the Hipparcos mission. Introducing objective prisms in front of the beam combiners will generate dispersed fringes which will allow a more accurate astrometry and much more detailed spectro-photometric information than filtered, undispered fringes. A complete optical system was designed which is suitable to achieve the desired goal and may be expanded to the size of GAIA. Likewise, several options exists to improve the capabilities and the depth of the DIVA mission. 